\title{
Frist Detection of The blaOXA-23 Genes in Clinical Isolates of Acinetobacterbaumanniiin Babylon Hospitals-Iraq
}

\author{
Raad A. AL-Harmoosh*, Eman M. Jarallah**, Anmar M. AL-Shamari***, \\ Hussein M.AL-khafaji****, Sura I. Jabuk**, Essam M. AL-Khaweledy* \\ * University of Kufa / College of Science, Najaf, IRAQ. \\ ** University of Babylon / College of Science, Babylon, IRAQ. \\ *** Ministry of Health and Environment/ Babylon Environment office / Babylon, IRAQ. \\ ****Ministry of Health and Environment/ Babylon Health office / Babylon, IRAQ.
}

\begin{abstract}
Acinetobacter spp. have risen as important pathogens in medicinal services related contaminations. Carbapenems are vital antimicrobial agents for treating diseases due to multidrug resistant Acinetobacter spp. Resistance to these drugs in the genus, may beconferred by different mechanisms particularly production of class D carbapenemases. OXA-23-like family has been brought up as one of the dominating carbapenemases among Acinetobacter. The present study aimed to investigate the occurrence of OXA-23-like carbapenemases among Acinetobacterisolatesrecovered from patients of hospitals in Babylon, Iraq. Antimicrobial susceptibility profiles were determined by disk-diffusion method.Imipenem resistant isolates were submitted to Modified Hodge Test and Double Disk Synergy Test to screen for carbapenemase generation, and later to polymerase chain reaction (PCR) to explore the presence of blaOXA-23 genes. One (10\%) of isolates was observed to be imipenem and meropenem resistant (MIC $>512 \mu \mathrm{g} / \mathrm{ml}) .6(60 \%)$ gave positive results with the imipenem-EDTA disk and Modified Hodge test ; 4 isolates (40\%) as indicated by PCR results, carried the blaOXA-23 genes. OXA-23-like enzymes might be a critical component of carbapenem resistance among isolates present in the hospitals studied.
\end{abstract}

Keywords: blaOXA-23, Acinetobacter, Oxacillinase, Carbapenem, MDR)

\section{Introduction}

Acinetobacter spp. have risen as a standout amongst the most essential pathogens involved in health care associated infections in late decades. These non-fermentative Gram-negative coco-bacilli are oftentimes involved in the etiology of ventilator related pneumonia, bacteremia, urinary tract diseases, and surgical site contaminations. These pathogens are additionally famous for their capacity to accumulate different mechanisms of antimicrobial resistance, frequently demonstrating a multidrug- resistant phenotype (1-3).

Carbapenems are viewed as critical antimicrobial agents for treating diseases due to multidrug-resistant Acinetobacter spp. In any case, reports of resistance to these medications have developed, with expanding recurrence, among Acinetobacter spp. clinical isolates (4-6).Different mechanisms may confer carbapenem resistance in Acinetobacter spp., yet creation of carbapenemases is viewed as the most vital one, especially those belonging to Ambler's class D, otherwise called oxacilinases (OXA), (7). The class B carbapenemases or metallo- $\beta$-lactamases (MBLs) can likewise be found among Acinetobacter spp., albeit less frequently (8).

Five major groups of OXA with carbapenemase activity have been distinguished in Acinetobacterbaumannii: involved OXA-23-like, OXA-40-like, OXA-58-like, and OXA-143-like families, and the OXA-51 group, which arranges a chromosomal oxacilinaseintrinsic for A. baumannii. Whenever overexpressed, these compounds can give carbapenem resistance.Among OXA, the variations involving the OXA-23-like family have been identified all through the world, and have also beenpointed out as the predominantcarbapenemases among Acinetobacter in a several geographic areas(9-11). The present study aimed to investigate the occurrence of isolates producing the OXA-23-like carbapenemases among imipenem and/or meropenem-resistantAcinetobacterisolates, isolated from Babylon hospitals-Iraq.

\section{Isolation and Identification of A. baumannii :}

\section{Materials And Methods}

A total of 1300 clinical specimens (included 588 burn swabs, 136 wound swabs, 50 from throat, 204 urine, 110 stool, 20 sputum, 162 blood, 15 ears and 15 eyes) were gathered from patients in Babylon Province hospitals more than one year time span beginning from March, 2014 to March, 2015. Isolates were recuperated from clinical specimens after culturing on MacConkey agar and incubated for overnight at $37^{\circ} \mathrm{C}$, lactose non fermenting bacteria were sub-cultured and incubated for extra overnight. Suspected bacterial isolates which 
theircells are Gram negative cocco-bacillary or diplo-bacillus and negative to oxidase which further distinguished utilizing API20 E system.

\section{Antimicrobial susceptibility testing:}

Isolates were cultured on Mueller-Hinton agar and their susceptibilities to various antibiotic agents were tested by disk diffusion method as indicated by the Clinical and Laboratory Standard Institute's rules (12).

\section{MIC determination:}

Contingent upon producer's guidelines the antibiotic stripes (E-test) were applied to the agar surface, the antibiotics promptly diffuses into the encompassing medium in high to low concentration from one end of the strip to the next. The gradient stays stable after dispersion, and the zone of inhibition made takes the form of oval (Liofilchemmanufacture). Furthermore micro-broth dilution method was done.

Imipenem-EDTA double disks synergy test:

Screening for metallo $\beta$-lactamases (MBL) was performed utilizing disks containing $1900 \mu \mathrm{g}$ of EDTA in addition to $10 \mu \mathrm{g}$ of imipenem disks were placed on the inoculated plates containing Muller Hinton agar. An expansion of $\geq 17 \mathrm{~mm}$ in zone diameter in the presence $1900 \mu \mathrm{g}$ of EDTA contrasted with imipenem alone showed the presence of a MBL (13).

Modified Hodge test:

Imipenem was utilized for carbapenemase detection as described by Lee and his colleagues (14). Positive test has a clover leaf-like indentation of $E$. coli Top-10 growing along the test organism growth streak inside the imipenem disk diffusion zone.

\section{Genotypic recognition of blaOXA-23 genes :}

DNA was extracted from the isolates by using genomic extraction mini kit according to the manufacture instructions (Bioneer company, Korea) . To amplify the genes encoding carbapenemases, a monoplex-PCR was run using the primers of blaOXA-23 (501bp: F/'5-GAT CGG ATT GGA GAA CCA GA3'and R/'5-ATT TCT GAC CGC ATT TCC AT-3') were described by Turton and his colleagues (15).

Amplification was performed in a $20 \mu \mathrm{l}$ volume as recommended by Promega Master mix instruction. PCR amplifications were carried out on a thermal cycler (Prime, England). The cycling conditions for amplification were as follows: initial denaturation of $94^{\circ} \mathrm{C}$ for $3 \mathrm{~min}$ and 30 cycles of $1 \mathrm{~min}$ at $94^{\circ} \mathrm{C}, 1 \mathrm{~min}$ at $57^{\circ} \mathrm{C}$, and $1 \mathrm{~min}$ at $72^{\circ} \mathrm{C}$, followed by $3 \mathrm{~min}$ at $72^{\circ} \mathrm{C}$. Amplified products were detected by agarose gel electrophoresis in $1 \%$ Tris-borate-EDTA (TBE) agarose (Promega, USA) and staining with ethidium bromide. The electrophoresis result was detected by using gel documentation system (Claver, England).

\section{Isolation and Identification of A. baumannii :}

\section{Results}

Depending on the biochemical tests and API20E system it has been able to isolate and identify of 10 $(0.76 \%)$ isolates as A. baumannii from the 1300 clinical samples (Table 1 ).

\section{Antimicrobial susceptibility testing :}

As determined by disk-diffusion method, every one of the A. baumanniiisolates showed distinctive pattern of resistance to various antibiotic agents (Fig.1), exhibiting highest resistance to penicillins (carbenicillin and ampicillin) with resistance rate of (100\%), while 3(30\%) of resistance were resistant to piperacillin. High resistance rates were watched for each of amoxicillin/clavulanic acid and aztreonam $(80 \%)$, (70\%) for cefepime , $(60 \%)$ for each of ceftazidime and cefotaxime.The results likewise revealed that were high resistance rates for each of tobramycine, and gentamicin (70\%) and moderate to amikacin (50\%). The isolates demonstrated low resistance rates for the carbapenem antibiotic agents, imipenem, meropenem and ertapenem (10\%). The percentage of resistance rate of the remaining antibiotic agents were as the following : (80\%) for chloramphenicol, followed by colistin sulfate with (70\%), polymyxin B (50\%), trimethoprim-sulfamethoxazole (50\%), (40\%) for quinolones, (ciprofloxacin) and (20\%) to each of tetracycline and doxycycline. Results revealed that all tested isolates were resistant at least of three classes of antibiotics, so that these isolates were considered to be multidrug resistant.

Table (1): Distribution of bacterial isolates recovered from clinical specimens among various hospitals in Babylon Province.

\begin{tabular}{|l|l|l|l|l|}
\hline Hospital's name & $\begin{array}{l}\text { No. of } \\
\text { samples }\end{array}$ & $\begin{array}{l}\text { No. (\%) of Acinetobacter } \\
\text { baumannii isolates }\end{array}$ & $\begin{array}{l}\text { No. (\%) of other No. (\%) of no } \\
\text { bacterial spp. isolates }\end{array}$ & $\begin{array}{l}\text { No. } \\
\text { growth cultures }\end{array}$ \\
\hline Al- Hillah Teaching Hospital & 885 & $7(0.8 \%)$ & $710(80 \%)$ & $168(18 \%)$ \\
\hline $\begin{array}{l}\text { Babylon Teaching Hospital } \\
\text { for Maternity and Pediatric }\end{array}$ & 415 & $3(0.7 \%)$ & $235(56.6 \%)$ & $177(42.6 \%)$ \\
\hline Total & 1300 & $10(0.76 \%)$ & $945(72.69 \%)$ & $345(26.5 \%)$ \\
\hline
\end{tabular}




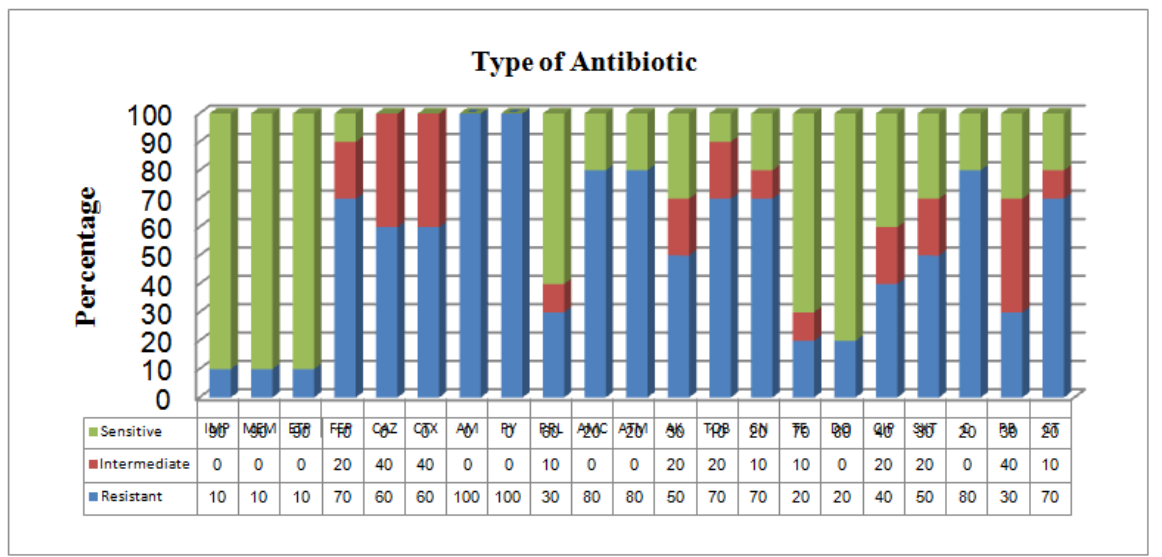

Figure (1) : Antibiotics susceptibility profile of A. baumannii isolates by disk diffusion method $(\mathrm{n}=10)$

(IMP,Imipenem ; MEM,Meropenem ; ETP,Ertapenem ; FEP,Cefepime ; CAZ,Ceftazidime ; CTX,Cefotaxime ; AM,Ampicillin ; PY,Carbenicillin ; PRL,Piperacillin ; AMC,Amoxi-clav; ATM,Aztreonam ; AK,Amikacin ; TOB,Tobramycine ; CN,Gantamicin ; TE,Tetracycline ; DO,Doxycycline ; CIP,Ciprofloxacin ; SXT,Trimethoprim-Sulfamethoxazole ; C,Chloramphenicol ; PB,Polymyxin B ; CT,Colistinsulphate).

\section{MIC determination:}

Table (2) revealed that $A$. baumannii isolates were resistant to imipenem, meropenem, and ertapenem with concentrations beyond values: $0.032 \mu \mathrm{g} / \mathrm{ml}$ - >512 $\mu \mathrm{g} / \mathrm{ml}$.

Table (2) : MIC of carbapenem antibiotics

\begin{tabular}{|l|l|l|l|}
\hline \multirow{2}{*}{ Isolates No. } & \multicolumn{4}{|l|}{ MIC $(\boldsymbol{\mu g} / \mathbf{m l})$} \\
\cline { 2 - 4 } & IMP & MER & ERT \\
\hline 1 & 0.75 & 0.032 & 0.032 \\
\hline 2 & 0.25 & 0.38 & 2 \\
\hline 3 & 1 & 0.047 & 0.023 \\
\hline 4 & 0.19 & 0.38 & 1.5 \\
\hline 5 & 0.38 & 1.5 & 6 \\
\hline 6 & $>512$ & $>512$ & $>512$ \\
\hline 7 & 0.19 & 0.75 & 3 \\
\hline 8 & 1 & 0.5 & 2 \\
\hline 9 & 1.9 & 0.047 & 0.032 \\
\hline 10 & 1 & 0.064 & 0.047 \\
\hline
\end{tabular}

\section{Phenotypic detection of carbapenem production:}

$1(10 \%)$ isolate exhibited upgrade of inhibition zone, with the imipenem-EDTA test whereas six isolates indicated positive results with modified Hodge test.

\section{Genotypic detection of blaOXA-23 genes :}

blaOXA-23genes were appeared in (40\%) of A. baumannii isolates PCR products using specific primers gene. (Fig. 2). Consequently, table (3) shows the isolates that harbored blaOXA-23 genes appeared as extensive drug resistant (XDR), which exhibited resistance to at least 5 classes of antibiotics were used in this study.

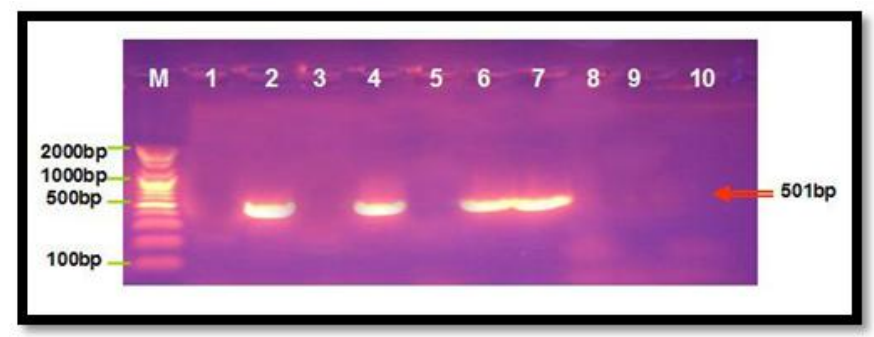

Figure (2):Agarose gel electrophoresis (1.5\% agarose,70 volt for 1-2 hrs) for blaOXA-23 gene product (amplified size 501bp). Lane (M), DNA molecular size marker (100- bp Ladder). Lanes (2, 4, 6, and 7 ) of $A$. baumannii isolates show positive results with blaOXA-23 gene. Lanes (1, 3, 5, 8, 9 and 10) show negative results with blaOXA-23 gene. 
Table (3) : Antibiotics resistant pattern of carbapenemase genes positive A. baumanniiisolates.

\begin{tabular}{|c|c|c|}
\hline Type of resistance & No. (\%) of $\boldsymbol{A}$. baumannii isolates (N=10) & No. of antibiotics classes resisted \\
\hline MDR & $10(100 \%)$ & 3 or more \\
\hline \multirow{3}{*}{ XDR } & $2(20 \%)$ & 9 \\
\cline { 2 - 3 } & $3(30 \%)$ & 7 \\
\cline { 2 - 3 } & $4(40 \%)$ & 6 \\
\cline { 2 - 3 } & $5(50 \%)$ & 5 \\
\hline PDR & $1(10 \%)$ & 11 \\
\hline
\end{tabular}

\section{Discussion}

The isolation rate of Acinetobacterbaumannii appeared with low percentage $(0.76 \%)$ accordingly to the biochemical tests and API20E. High resistance rates were observed for most of the antimicrobials agents studied, including, penicillins, amoxicillin/clavulanic acid, aztreonam, and chloramphenicol. Antimicrobial resistance considerably restricts the available treatment options, especially resistance to carbapenem, which is considered to be the first option to treat severe infections due to Acinetobacter spp.(16). El-Astal, mentioned that inappropriate and incorrect administration of antimicrobial agents and lack of appropriate infection control strategies may be the possible reasons behind increasing resistant rate of A. baumannii to common used antimicrobial drugs (17).

All $A$. baumannii isolates were screened by two phenotypic tests for carbapenemase production. The present study showed that $(10 \%)$ of the isolates gave positive results by imipenem- EDTA disk test . Different studies which have used the IMP-EDTA to detect MBLs production in A. baumannii reported that (33\%) of isolates have enhancement of inhibition zone, with the IMP-EDTA test (18). However, there are four isolates which gave negative results with EDTA disk synergy test.

The most easily performed test for Carbapenemase detection is the modified Hodge's test, which has been found to be $100 \%$ sensitive for the detection of the carbapenemase (14). Out of the 10 A. baumannii isolates which were enrolled in this study, $6(60 \%)$ isolates were found to produce the carbapenemase enzyme by MHT and all the remaining isolates were found to be carbapenemase negative . In a previous local study, Alsehlawi and hiscolleagues reported that $4(33.3 \%)$ of A. baumannii isolates recovered from Najaf hospitals were confirmed as carbapenemase producer using modified Hodge's test, whereas the same isolate gave negative result with imipenem-EDTA synergy test (18). Another study from Croatia the Hodge test showed that 74\% (72/97) of the A. baumannii isolates were positive for carbapenemase production (19), whereas in a study from Pakistan has shown that $17 \%$ of A. baumannii were positive for carbapenemase production by MHT (20).

Oxacillinases are only weakly active against carbapenems and are largely confined to Pseudomonas and Acinetobacter species and only rarely in Enterobacteriaceae (21). blaOXA-23 represented a new subset of the OXA family. It has been identified in outbreaks of carbapenem-resistant Acinetobacter in Brazil, Korea and United Kingdom $(15,22,23,24)$. Result from present study show that 4(40\%) A. baumannii isolates had blaOXA-23 genes (Fig. 2) . In the study in Iraq, Alsehlawi, andhiscolleagues who found 2(40\%) A. baumannii isolates had blaOXA-23 genes positive in Najaf hospitals (18). In contrast in Taiwan study, Lee, and hiscolleagues documented only one isolate (4\%) of A. baumannii was harbored blaOXA-23 gene (25).

The isolates blaOXA-23 positive A. baumannii exhibit resistance to most tested antimicrobials and appeared to be MDR (Table 3 this may making a significant issue for decision of treatment, this results was more indistinguishable with the report of emergence XDR in A. baumannii isolates from patients in ICUs of Samsung Medical Center in Seoul, South Korea (26). Subsequently, The event of isolates contain blaOXA-23 in Babylon Province hospitals may came from because of exchange of plasmid among resistant isolates, a few isolates may create indistinguishable restriction pattern resulting of blaOXA-23 dissemination because of a clonal spread of resistant A. baumannii isolates (27).

\section{Conclusion}

Our study has demonstrated low spreading rate of multidrug resistant and blaOXA-23 harbored $A$. baumannii isolates among patients with various infections. Sadly, numerous antibiotics endorsed to individuals are superfluous. As well as the overuse and misuse of antibiotics helps to produce drug-resistant bacteria.

\section{Acknowledgements}

The authors are exceptionally thankful to department of biology in faculty of science and the laboratory staff of Al- Hillah Teaching Hospital and Babylon Teaching Hospital for Maternity and Pediatric.The authors thank the unknown reviewers for their significant remarks and accommodating correction recommendations.

\section{References}

[1]. Fishbain, J. and Peleg, A.Y.(2010). Treatment of Acinetobacter Infections. Cli. Infect. Dis. 51:79-84.

[2]. Omran, L. and Askar, E. (2016). Antibiotic Sensitivity Patterns of the Most Common Bacteria Isolated from Al-Mouwasat University Hospital in 2015,Syria. International Journal of PharmTech Research.9 : (1) 113-119. 
[3]. Gordon, N.C. and Wareham, D.W.(2010). Multidrug-resistant Acinetobacterbaumannii: mechanisms of virulence and resistance. Int J Antimicrob Agents. 35:219-26.

[4]. Gaur, A. ; Garg, A. ; Prakash, P. ; Anupurba, S. and Mohapatra, T.M. (2008) . Observations on carbapenem resistance by minimum inhibitory concentration in nosocomial isolates of Acinetobacter species: an experience at a tertiary care hospital in north India. J Health PopulNutr. 26:183-8.

[5]. Towner, K.J. (2009). Acinetobacter: an old friend, but a new enemy. J Hosp Infect. 73:355-63.

[6]. Machado, G.M. ; Lago, A. ; Fuentefria, S.R. and Fuentefria, D.B. (2011). Occurrence and the susceptibility to antimicrobial agents in Pseudomonasaeruginosa and Acinetobacter spp. at a tertiary hospital in southern Brazil. Rev Soc Bras Med Trop. 44:168-72.

[7]. 7-Saravanan, D. and Radhakrishnan, M.(2016). Antimicrobial activity of mangrove leaves against drug resistant pathogens.International Journal of PharmTech Research. 9 (1) : 141-146.

[8]. Gussati, C.S. ; Ferreira, A.S. ; Fuentefria, D.B. and Corc, G. (2009). Resistência aß-lactâmicosemAcinetobacter spp. isolados de efluentehospitalar no sul do Brasil. Rev Soc Bras Med Trop. 42:183-7.

[9]. Higgins, P.G. ; Poirel, L. ; Lehmann, M. ; Nordmann, P. and Seifert, H.(2009). OXA-143, a novel carbapenem-hydrolyzing class D beta-lactamase in Acinetobacterbaumannii. Antimicrob Agents Chemother. 53:5035-8.

[10]. Poirel, L. and Nordmann, P. (2006). Carbapenem resistance in Acinetobacterbaumannii: mechanism and epidemiology. ClinMicrobiol and Infec. 12:826-836.

[11]. Walsh, T.R. (2010). Emerging carbapenemases: a global perspective. Int J Antimicrob Agents.36Suppl3:S8-14.

[12]. 12-Clinical and Laboratory Standards Institute. (2012). Performance standards for antimicrobial susceptibility testing: 22nd informational supplement. CLSI document M100-S22. CLSI, Wayne, PA.

[13]. 13-Lee, K. ; Lim, Y.S. ; Yong, D. ; Yum, J. H. and Chong, Y. (2003). Evaluation of the Hodge test and the imipenem-EDTA double-disk synergy test for differentiating metallo- $\beta$-lactamase-producing isolates of Pseudomonas spp. And Acinetobacter spp. J. Clin. Microbiol. 41(10), 4623-4629.

[14]. 14-Lee, K., Chong, Y., Shin, H. B., kim, Y.A ., Yong, D. and Yum, J. H. (2001). Modified Hodge and EDTA-disc synergy tests to screen metallo- $\beta$-lactamase-producing strains of Pseudomonas and Acinetobacter species .Clin. Microbiol .Infect. 7: 88-91.

[15]. 15-Turton, J. F. ; Woodford, N. ; Glover, J. ; Yarde, S. ; Kaufmann, M. E. ; and Pitt, T. L.(2006). Identification of Acinetobacter baumannii by Detection of the blaOXA-51-like Carbapenemase Gene Intrinsic to This Species. Journal of clinical microbiology .44 (8): 2974-2976.

[16]. 16-Corrêa, L. L. ; Botelho, L. A. B. ; Barbosa, L. C.; Mattos, C. S. ; Carballido, J. M. ; Castro, C. L.T. ; Mondino, P. J. J., de Paula, G. R, de Mondino, S. S. B. ; de Mendonca-Souza, C. R.V. (2012). Detection of blaOXA-23 in Acinetobacter spp. isolated from patients of a university hospital. Braz j infect dis. 16(6):521-526.

[17]. 17-El-Astal, Z. (2005). Increasing ciprofloxacin resistance among prevalent urinary tract bacterial isolates in Gaza Strip, Palestine. J. Biomed Biotechnol. (3) : 238-241.

[18]. 18-Alsehlawi, Z. S. ; Alshara, J. M. ; Hadi, Z.J. ; Almohana, A. M. (2014). First report of the ${ }^{b l a}$ oxa-23 gene in a clinical isolates of Acinetobacter baumannii in Najaf hospitals-Iraq. Int. J. Recent Sci. Research. 5(8) : 1407-1411.

[19]. 19-Firoozeh, F. ; Zibaei, M. and Soleimani-As, Y. (2014). Detection of plasmid-mediated qnr genes among the quinolone-resistant Escherichiacoli isolates in Iran. J. Infect. Dev. Ctries. 8(7): 818-822.

[20]. 20-Amjad, A. ; Mirza, I.A. ; Abbasi, S.A. ; Farwa, U. ; Malik, N. and Zia, F. (2011). Modified Hodge test: A simple and effective test for detection of carbapenemase production. Iran J. Microbiol. 3(4) :189-193.

[21]. 21-Walther-Rasmussen, J. and Hoiby, N.(2006). OXA-type carbapenemases. J. Antimicrob. Chemother. 57: 373-383.

[22]. 22-Dalla-Costa L.M. ; Coelho J.M. ; Souza H.A. ; Castro M.E. ; Stier C.J. ; Bragagnolo K.L. ; Rea-Neto A. ; Penteado-Filho S.R. ; Livermore D.M. and Woodford, N.(2003). Outbreak of carbapenem-resistant Acinetobacterbaumannii producing the OXA-23 enzyme in Curitiba, Brazil. J. Clin. Microbiol., 41: 3403-3406.

[23]. 23-Jeon, B-C. ; Jeong, S. H. ; Bae, I. K. ; Know, S. B. ; Lee, K. ; Young, D. ; Lee, J.H. ; Song. J.S. and Lee, S.H. (2005). Investigation of a nosocomial outbreak of imipenem-resistant Acinetobacter baumannii producing the OXA-23 $\beta$-lactamase in Korea. J. Clin. Microbiol. 43(5): 2241-2245.

[24]. 24-Stoeva, T. ; Higgins, P.G. ; Bojkova, K. and Seifert, H. (2008). Clonal spread of carbapenem resistant OXA-23-positive Acinetobacter baumannii in a Bulgarian university hospital. Clin. Microbiol. Infect. 14:723-727.

[25]. 25-Lee, M.H. ; Chen TL, Lee YT, Huang L, Kuo SC, Yu KW, Hsueh PR, Dou HY, Su IJ, Fung CP. (2013). Dissemination of multidrug resistant Acinetobacter baumannii carrying blaOXA-23 from hospitals in central Taiwan. J Microbiology, Immunology and Infection. 46(6):419-24.

[26]. 26-Kim, K.M. ; Yoo, J.H. ; Choi, J.H. ; Park, E.S. ; Kim, K.S. and Kim, K.S.(2006). The nationwide surveillance results of nosocomial infections along with antimicrobial resistance in intensive care units of sixteen university hospitals in Korea. Kor., J. Noso. Infect. Control, 11:79-86.

[27]. 27-Chang, K., Lin, M., Lin, N., Wu, W., Kuo, H., Lin, T., Yang, T., Chen, Y. and Liou, M. (2012). Clonal spread of multidrugresistant Acinetobacterbaumannii in eastern Taiwan. J. Microbiology, Immunology and Infection.45: 37-42. 